\title{
Stable Prediction of Mood and Anxiety Disorders Based on Behavioral and Emotional Problems in Childhood: A 14-Year Follow-Up During Childhood, Adolescence, and Young Adulthood
}

Sabine J. Roza, M.Sc.

Marijke B. Hofstra, M.D.

Jan van der Ende, M.S.

Frank C. Verhulst, M.D.
Objective: The goal of this study was to predict the onset of mood and anxiety disorders from parent-reported emotional and behavioral problems in childhood across a 14-year period from childhood into young adulthood.

Method: In 1983, parent reports of behavioral and emotional problems were obtained with the Child Behavior Checklist for children and adolescents 4-16 years of age from the Dutch general population. At follow-up 14 years later, lifetime mood and anxiety diagnoses were obtained by a standardized DSM-IV interview for 1,580 subjects. Cox proportional hazards models were used to predict the incidence of mood and anxiety disorders from childhood problems and demographic covariates.

Results: Mood disorders were significantly predicted by high scores on the anxious/depressed scale and on the inter- nalizing composite (withdrawn, somatic complaints, and anxious/depressed). Anxiety disorders were significantly predicted by the social problems scale and the externalizing composite (delinquent behavior and aggressive behavior). Anxiety disorders predominantly started in childhood and early adolescence, whereas the incidence of mood disorders increased sharply in adolescence and young adulthood.

Conclusions: These results suggest different developmental pathways for mood and anxiety disorders. The predictions based on problem behavior remained stable during the 14-year period across adolescence and young adulthood. The results therefore underline the importance of early intervention and prevention of behavioral and emotional problems in childhood.

(Am J Psychiatry 2003; 160:2116-2121)

$\mathrm{T}$ he epidemiology of anxiety and mood disorders, in adults as well as in adolescents, has been studied over the past two decades by using standardized diagnostic instruments such as the National Institute of Mental Health Diagnostic Interview Schedule (1) or the Composite International Diagnostic Interview (2). These studies (for example, those described in references 3-6) have shown that anxiety and mood disorders are frequently occurring psychiatric disorders, with lifetime prevalences between 19\% and $28 \%$. Both disorders are much more frequent in females than in males.

In recent years, more attention has been paid to the distributions of the ages at onset of the different disorders. Anxiety disorders predominately start in childhood or early adolescence, whereas depressive disorders are associated with increasing incidences in late adolescence and early adulthood (7). These distributions are similar in males and females. However, the causes for these different onsets are unknown. Finding predictors of the age at onset of mood and anxiety disorders would be an important step in unraveling these causes.
Several researchers have described associations between emotional and behavioral problems in childhood and psychiatric disorders in adulthood. Behaviorally inhibited children are more likely to meet diagnostic criteria for depression in adulthood, according to data adjusted for gender $(7,8)$. Rule-breaking behavior in childhood predicts adult anxiety disorders in women and mood disorders in men (9). The associations found in these studies were presented as odds ratios, reflecting the presence of significant associations between childhood behavior and adult psychiatric disorders. To address questions about whether and, particularly, when mood and anxiety disorders develop, other statistical techniques are needed (10).

Our study concerns a 14-year follow-up of parent-reported behavioral and emotional problems, determined in 1983 with the Child Behavior Checklist (11), in subjects randomly selected from the Dutch general population, initially 4-16 years of age. In 1997, mood and anxiety diagnoses were retrospectively obtained with the Composite International Diagnostic Interview. The main aims of the present study were 1) to examine temporal patterns in the 
onset of mood and anxiety disorders, 2) to predict the onset of mood and anxiety disorders from parent-reported emotional and behavioral problems across a 14 -year period during adolescence and young adulthood, 3) to evaluate the stability and change of the strength of the prediction across time, and 4) to test whether gender, age at initial assessment, and socioeconomic status are related to the likelihood of developing mood and anxiety disorders, after the problem behavior is accounted for, and whether these relationships change across time.

\section{Method}

\section{Sample}

The original sample of 2,600 children and adolescents 4-16 years of age was drawn from the Dutch province of Zuid-Holland in 1983. A random sample of 2,600 children and adolescents (100 children of each sex and each birth year cohort) was drawn from municipal registers that list all residents in the province. Two small municipalities out of a total of 86 declined to participate. Of the 2,447 parents who were reached, 2,076 (84.8\%) cooperated by completing a Child Behavior Checklist (11) on their child $(12,13)$. This sample was followed up at 2-year intervals until 1991. In 1997 the sample was approached again, for the sixth time. More details on the initial data collection are presented elsewhere $(12,13)$. In $1997,1,580$ of the 2,076 subjects at initial assessment provided information for determining DSM-IV diagnoses. This information was obtained by using the computerized version of the Composite International Diagnostic Interview (2). Corrected for deceased $(\mathrm{N}=8)$, mentally retarded $(\mathrm{N}=12)$, and emigrated $(\mathrm{N}=59)$ subjects, the response rate was $79.1 \%$. The other 419 subjects for whom no DSM-IV information was obtained ("dropouts") had 1983 Child Behavior Checklist total problems scores similar to those of the subjects who remained $(\mathrm{N}=1,580)$. Because of the selection of DSM-IV diagnoses, the sample differs slightly from that reported earlier (9). The Dutch human subjects committee approved this study, and written informed consent was obtained from all subjects after the procedures had been fully explained.

\section{Instruments}

The Child Behavior Checklist is a questionnaire to be completed by parents of 4 - to 18 -year-olds. The problem section contains 120 items on behavioral and emotional problems during the past 6 months, scored $0=$ nontrue, $1=$ somewhat or sometimes true, and 2=very true or often true. The Child Behavior Checklist can be scored for eight syndromes: withdrawn, somatic complaints, anxious/depressed, social problems, thought problems, attention problems, delinquent behavior, and aggressive behavior. These syndromes can be grouped in two broad scales: internalizing encompasses the first three syndromes; externalizing encompasses the last two. Summing the individual item scores provides a total problems score. Good reliability and validity of the Child Behavior Checklist have been replicated for the Dutch translation (14).

The Composite International Diagnostic Interview contains approximately 300 questions covering DSM-IV criteria. Because the cell sizes for specific disorders were small for the majority of diagnoses, we constructed two main groupings of DSM-IV categories. Anxiety disorders consisted of generalized anxiety disorder, obsessive-compulsive disorder, panic disorder, agoraphobia, social phobia, specific phobias, posttraumatic stress disorder, or any combination of these disorders. Mood disorders were major depressive episode, bipolar disorder, dysthymia, or any combina- tion of these disorders. Good reliability and validity have been reported for the Composite International Diagnostic Interview (15).

Socioeconomic status of the parents at time 1 was measured by using a 6-point scale of parental occupation (16) with 1 as lowest socioeconomic status and 6 as highest. If both parents worked, the higher occupational level was used.

\section{Statistical Analysis}

The cumulative incidences of the two types of disorders were determined by using life tables. Survival time was defined as age at onset of the psychiatric disorder.

The hazard ratios reported, which describe the association between childhood problems and adult disorders, were computed by using Cox continuous-time proportional hazards models (17). Survival time was defined as years between initial administration of the Child Behavior Checklist and first complaints of psychiatric disorder. The diagnoses of mood and anxiety disorders as determined by the Composite International Diagnostic Interview were the events to be predicted. We included the Child Behavior Checklist total problems score and the scores for internalizing, externalizing, and the eight problem scales as predictors of mood and anxiety disorders. The scores were dichotomized as deviant versus nondeviant. To select individuals who could be regarded as deviant at time 1 on the basis of their Child Behavior Checklist scores, the cutoff points recommended by Achenbach (11) were used. The cutoff points for the externalizing, internalizing, and total problem scores were the 82nd percentile of the cumulative frequency distributions of these scales for a Dutch normative sample (14), and for the syndrome scales they were the 95th percentile.

For each covariate, we tested the proportional hazards assumption by adding to the model an interaction with time, as recommended by Singer and Willett (18). If this improved the fit, the interaction was included in the model.

Age at initial Child Behavior Checklist administration, gender, and socioeconomic status were the covariates tested in the different models. Age was dichotomized (4-11 versus $12-16$ years) to test the differences between preadolescent children and adolescents. Parental socioeconomic status was also dichotomized as 1 , representing low status (ratings of 1,2, and 3), versus 2, for high status (ratings of 4,5 , and 6 ). For each covariate the possible interaction with the problem scores was tested.

We used the Bonferroni method to correct for multiple comparisons. Findings with $\mathrm{p}<0.05$ (two-tailed) were considered statistically significant. All statistical tests were performed by means of SPSS 9.0 for Windows (19).

\section{Results}

\section{Temporal Patterns}

Figure 1 shows the cumulative incidence of anxiety and mood disorders by age at onset for each gender separately. Cumulative incidence is the number of subjects developing a disorder during a certain time period divided by the total number of subjects followed for that period. The risk for onset of mood disorder sharply rose approximately beginning at age 13, with a steady increase up to age 30 . While the difference in risk between men and women was small in childhood up to the age of 12 years, in adolescence and young adulthood the risk difference between the two genders became larger.

Anxiety disorders were more frequent than mood disorders until the age of 25, both in males and females. After the age of 25 , the cumulative incidence of anxiety disor- 
FIGURE 1. Cumulative Incidence of Mood and Anxiety Disorders, by Age at Onset, in a Sample $(N=1,580)$ of Males and Females From the Dutch General Population First Assessed at Age 4-16 Years and Followed Up 14 Years Later

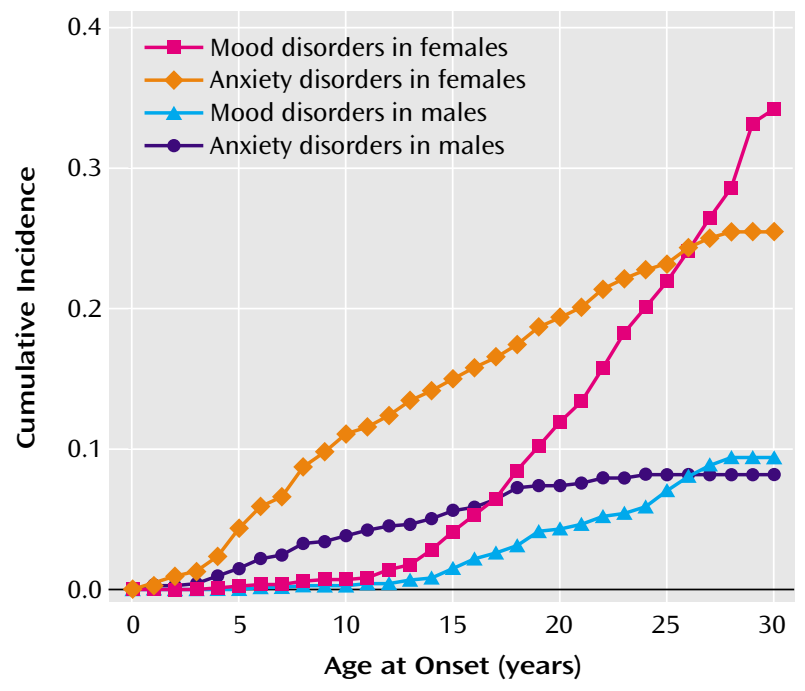

ders did not increase anymore, in contrast to the cumulative incidence of mood disorders.

For individuals who developed both mood and anxiety disorders during their lifetimes, the anxiety disorder started before the mood disorder in most cases $(68.8 \%, 64$ of 93). These 64 represent $27.6 \%$ of the 232 subjects with mood disorders. Only $10.8 \%(\mathrm{~N}=10)$ of the 93 subjects with both disorders developed the mood disorder before the anxiety disorder. The time between the onsets of the two disorders was significantly longer for the subjects who developed the anxiety disorder first; the mean for this group was 9.2 years, and the $95 \%$ confidence interval (CI) was 7.8-10.6, whereas for those whose mood disorder was diagnosed first, the mean was 2.2 years $(95 \% \mathrm{CI}=1.1-3.3)$. In 19 subjects $(20.4 \%)$, the anxiety disorder and the mood disorder started within the same year.

Of the 232 subjects with mood disorders, major depressive disorder was the most frequent diagnosis $(92.7 \%, \mathrm{~N}=$ 215). The other mood disorders were less common; bipolar disorder was diagnosed in $5.2 \%(\mathrm{~N}=12)$, and dysthymia was diagnosed in $2.6 \%$ ( $\mathrm{N}=6$ ). Among the 250 subjects with anxiety disorders, the most frequent diagnoses were specific phobia (57.6\%, $\mathrm{N}=144)$, posttraumatic stress disorder (18.8\%, $\mathrm{N}=47)$, and social phobia $(12.8 \%, \mathrm{~N}=32)$.

\section{Predictors of Mood Disorders}

Of the 1,580 subjects for whom the Child Behavior Checklist was completed at initial assessment and a psychiatric interview was completed at follow-up, eight $(0.5 \%)$ subjects claimed to have complaints of mood disorder at the initial assessment and were excluded from the analyses to ensure that only new cases were considered in our analyses. The remaining 1,572 subjects formed the initial group "at risk."
The statistically significant estimates of the relative risk of mood disorders obtained from the Cox multiple regression analyses are given in Table 1 (left side). First, we tested eight models, all including one Child Behavior Checklist syndrome along with the covariates common to all models, i.e., gender and age. To correct for multiple comparisons, we used the Bonferroni method to adjust the $p$ values. The $p$ value obtained from each of the eight tests was multiplied by 8 . When the corrected $p$ value was smaller than 0.05 , the scale was a significant predictor. Anxious/depressed was the only syndrome that significantly predicted mood disorders. In the multivariate model, containing all moderately significant determinants $(\mathrm{p}<0.20)$, only anxious/depressed remained significant, indicating that this scale predicted the onset of mood disorders independent of the other Child Behavior Checklist scales. Adding the interaction with time to the model did not improve the model fit significantly.

The same procedure was applied to the broad scales for internalizing and externalizing. First, we adjusted each scale separately for the covariates age and gender. The $\mathrm{p}$ value obtained from each of these two tests was multiplied by 2 to correct for multiple comparisons. These analyses yielded a relative risk that was significant only for the internalizing scale. When both internalizing and externalizing (both moderately significant predictors of mood disorder, with $\mathrm{p}<0.20$ ) were included in one model, internalizing was not significant anymore, indicating that internalizing behavior did not predict the incidence of mood disorders independent of the prediction of externalizing behavior.

Gender and age significantly predicted mood disorders. Males were less likely to have a mood disorder in the follow-up period than females. Younger children (4-11 years of age) were less likely to have a mood disorder than older children (12-16 years), but the relative risk decreased with the length of follow-up. Socioeconomic status was not related to the onset of mood disorders.

There were no significant interactions between the problem scales and the demographic covariates, i.e., the associations between problem behavior and mood disorders were similar for males and females, for ages 4-11 and 12-16 years, and for the two levels of socioeconomic status.

Results regarding the covariates age and gender were similar across the models. Table 1 shows the estimates of age and gender in the multivariate analysis.

\section{Predictors of Anxiety Disorders}

Of the 1,580 subjects, 106 (6.7\%) claimed to have complaints of anxiety disorder at the initial assessment and were excluded from the analyses to ensure that only new cases were considered. The remaining 1,474 subjects formed the initial group "at risk."

The significant estimates of the relative risk of anxiety disorders obtained from the Cox multiple regression analyses are given in Table 1 (right side). Again, we tested eight 
TABLE 1. Significant Results of Cox Multiple Regression Analyses of the Risk of Mood and Anxiety Disorders in a Sample From the Dutch General Population First Assessed at Age 4-16 Years and Followed Up 14 Years Later

\begin{tabular}{|c|c|c|c|c|c|c|}
\hline \multirow[b]{2}{*}{ Variable } & \multicolumn{3}{|c|}{ Mood Disorders $(\mathrm{N}=232)$} & \multicolumn{3}{|c|}{ Anxiety Disorders $(\mathrm{N}=250)$} \\
\hline & Hazard Ratio & $95 \% \mathrm{Cl}$ & $\mathrm{p}$ & Hazard Ratio & $95 \% \mathrm{Cl}$ & $\mathrm{p}$ \\
\hline \multicolumn{7}{|l|}{ Child Behavior Checklist $^{a}$} \\
\hline \multicolumn{7}{|l|}{ Syndrome scales } \\
\hline Anxious/depressed & $1.87^{b}$ & & 0.006 & & & \\
\hline Social problems & & & & $2.45^{b}$ & & 0.005 \\
\hline \multicolumn{7}{|l|}{ Summary scales } \\
\hline Internalizing & 1.44 & & 0.03 & & & \\
\hline Externalizing & & & & $1.80^{\mathrm{b}}$ & & 0.002 \\
\hline Total problems & & & & 2.05 & & 0.0001 \\
\hline \multicolumn{7}{|l|}{ Covariates } \\
\hline Male genderc & 0.28 & $0.20-0.38$ & & 0.31 & $0.21-0.45$ & \\
\hline Age $4-11$ years $^{d}$ & 0.23 & $0.10-0.53$ & & 1.56 & $1.07-2.28$ & \\
\hline By time & $0.90^{\mathrm{e}}$ & $0.83-0.97$ & & & & \\
\hline
\end{tabular}

a Scores were dichotomized as 0 (normal range) or 1 (borderline or clinical range).

${ }^{\mathrm{b}}$ Remained significant when entered into a model with other moderately significant predictors $(\mathrm{p}<0.20)$.

${ }^{\mathrm{c}} 0=$ female, $1=$ male.

d $0=12-16$ years old, $1=4-11$ years old

e Relative risk decreased with time.

models, each including one Child Behavior Checklist syndrome along with the covariates common to all models, i.e., gender and age. The $\mathrm{p}$ values were multiplied by 8 to correct for multiple comparisons. Only social problems was significant, indicating that only this syndrome had a significant relationship with the incidence of anxiety disorders independent of the other syndromes. Adding the interaction with time did not significantly improve the model fit.

When adjusted for age and gender, the externalizing scale was a significant predictor of the incidence of anxiety disorders. Externalizing also predicted the onset of anxiety disorders independent of internalizing behavior.

Gender and age significantly predicted anxiety disorders. Males were less likely to have an anxiety disorder in the 14-year follow-up. Children ages 4-11 years were more likely to have an anxiety disorder in the next 14 years than older children (12-16 years).

There were no significant interactions between the scales for problem behavior and the covariates age, gender, and socioeconomic status.

Results regarding the covariates age and gender were similar across the models. Socioeconomic status was excluded from the multiple models because its contribution to the prediction was not significant.

\section{Discussion}

The present study showed that emotional and behavioral problems reported by parents in childhood or adolescence on the Child Behavior Checklist predicted the onset of DSM-IV mood and anxiety disorders across a 14year period. None of the predictions based on problem behavior significantly changed across the follow-up time. Gender and age at initial assessment were significantly associated with the onset of mood and anxiety disorders. The relationship between age at initial assessment and the onset of mood disorders significantly changed across time. The subjects who were adolescents (12-16 years) at the initial assessment were more likely to have mood disorders than younger children (4-11 years). With longer follow-up time, when the subjects who were young children at the initial assessment grew up into teenagers, the relative risk decreased.

Like findings in other studies (5-7), our results showed that anxiety disorders predominantly started in childhood and early adolescence, whereas the incidence of depressive disorder increased sharply in adolescence and young adulthood. Both anxiety and mood disorders were more frequent in females than in males. In most individuals diagnosed with both anxiety and depressive disorders, the anxiety disorder started before the mood disorder.

\section{Predictors of Mood Disorders}

Mood disorders were significantly predicted by scores on the anxious/depressed scale of the Child Behavior Checklist. The broad internalizing scale, consisting of the withdrawn, somatic complaints, and anxious/depressed syndromes, predicted the onset of mood disorders but not independent of the score on the externalizing scale. Earlier reports showed findings similar to ours in that inhibited children were more likely to be diagnosed with mood disorders in adolescence and young adulthood (6, 8). However, some found this association to occur only in males (9), whereas others did not find significant interactions with sex (8). As in the current study, both males and females experienced higher risk for depression if they were anxious or depressed in childhood. It has been hypothesized (20) that anxious or inhibited temperament can increase stress reactivity. Especially in girls, the transition from childhood into adulthood during adolescence, with the physical changes related to pubertal development and the intensification of stereotypical gender roles, is experienced in a negative way (21). Our results show that both boys and girls with problems reflecting inhibited temperament are more vulnerable to later depression. 
However, girls are more likely to develop a depressive disorder, possibly because they experience more stress during the adolescent transition.

The strength of the prediction based on problem behavior showed stability across time, indicating that children who are anxious/depressed have a higher risk across the whole pubertal period and young adulthood than do "normal" children without emotional or behavioral problems, even 14 years after initial assessment.

\section{Predictors of Anxiety Disorders}

Scores for social problems significantly predicted anxiety disorders. Externalizing behavior significantly predicted the onset of anxiety disorders, independent of the internalizing score. Earlier reports showed these associations only in females (9), while our results were not conditioned by sex. Although the social problems scale, comprising items such as "acts too young for age," "too dependent," "does not get along with other kids," "gets teased a lot," "not liked by other kids," "poorly coordinated or clumsy," and "prefers being with younger kids," does not seem to have a clear counterpart in DSM-IV (22), our results showed a strong association between this scale and later anxiety disorders. A study among schoolchildren in the United Kingdom (23) demonstrated an association between being bullied at school and a high anxiety score based on self-reports. The downward spiral starting with poor social skills and difficulties in peer relationships, which can lead to low selfconfidence, negative self-evaluation, and self-criticism, leading to even more difficulties in peer relationships, up to being bullied and teased, can result in specific anxiety symptoms such as perceived danger and threat, uncertainty, and hypervigilance.

As reflected in the stability of the predictions found in the present study, the association between social problems and anxiety disorders does not decrease across time.

\section{Limitations}

The strengths of this study are its long follow-up period, spanning 14 years through adolescence and young adulthood, and its sample size, which was large enough to examine relatively common psychiatric disorders, such as major depressive disorder and several anxiety disorders. However, some potential methodological limitations need to be considered. As in other longitudinal studies, the generalizability is questionable because of sample attrition, despite the facts that we traced $79.0 \%$ of the time 1 sample and that our data did not suggest selective attrition. Second, the retrospective diagnoses of mood and anxiety disorders based on patients' reports of when they first experienced complaints of the disorders could have introduced recall error. People tend to underestimate past morbidity, which may lead to significant underestimation of the lifetime prevalences reported (24). Furthermore, respondents may "telescope" time and shift the age at first onset toward recent years, which would lead to overestimation of disease incidence during the period immediately before assessment with the Composite International Diagnostic Interview. This could explain the fact that we did not find significant changes in the predictions of mood and anxiety disorders across time. Because stability of recall is also related to receipt of treatment and because interventions could have influenced the course of problem behaviors from childhood into adulthood, a third limitation of the current study is the fact that we did not include standardized information on treatment during the follow-up period.

\section{Conclusions and Implications}

Despite the fact that almost $28 \%$ of the mood disorders were preceded by an anxiety disorder, our results suggest different developmental pathways for anxiety and mood disorders. Mood disorders in adulthood are generally predicted by parent-reported internalizing behavior, while anxiety disorders are predicted by parent-reported social problems and externalizing behavior in childhood. These predictions based on parent-reported problem behavior were not liable to recall biases. Supplementary to the discussion of whether anxiety and mood disorders are distinct psychiatric disorders, our results suggest that the two disorders have diverse roots in childhood. Speculations about a common pathophysiological mechanism for anxiety and depression (25) are therefore not supported by our results.

Gender differences were lacking in the predictions, indicating that the higher frequencies of both anxiety and mood disorders among females are due to factors other than problem behavior in childhood, such as environmental factors in adolescence or genes that come into expression later in life.

Even more striking about our results is the stability of the predictions during a 14-year period across adolescence and young adulthood.

Therefore, our results have several clinical implications, both for mental health professionals working with children and adolescents and for adult psychiatrists. The long-term consequences of problem behavior and emotional problems in childhood for later adult functioning support the importance of early intervention and prevention of such problems in young children. Parental reports are the most commonly used approach in studies of children ages 12 and under. Also, in the clinical evaluation of young children, parent reports might contribute to treatment planning and prognostic statements. For young children with externalizing behavior, the focus should be not only the disruptive aspects of their psychopathology but also their inner life and emotional needs. Moreover, the information on childhood origins of adult psychopathology adds to the general understanding of psychiatric disorders and may contribute to advances in diagnosis, treatment, and prognosis in adult psychiatry. 
Nonetheless, the overlap between anxiety and depressive symptoms, both within episodes and over a lifetime, remains unclear. At the same time, little is known about the developmental route of social problems into anxiety disorder. Further research is needed to understand the causal pathways of specific childhood behavior through anxiety disorders into major depressive disorder.

Received July 8, 2002; revision received Jan. 22, 2003; accepted March 2, 2003. From the Department of Child and Adolescent Psychiatry, Sophia Children's Hospital, Erasmus Medical Center. Address reprint requests to Dr. Verhulst, Department of Child and Adolescent Psychiatry, Sophia Children's Hospital, Erasmus Medical Center, Dr. Molewaterplein 60, 3015 GJ Rotterdam, the Netherlands; f.verhulst@ erasmusmc.nl.

Supported by grant 002827230 from the Health Research and Development Council (Zorgonderzoek, the Netherlands).

\section{References}

1. Robins LN, Cottler L, Bucholtz K, Compton W: National Institute of Mental Health Diagnostic Interview Schedule, version IV (DIS-IV). St Louis, Washington University, Department of Psychiatry, 1997

2. World Health Organization: Composite International Diagnostic Interview (CIDI), version 1.0. Geneva, WHO, 1990

3. Kessler RC, McGonagle KA, Zhao S, Nelson CB, Hughes M, Eshleman S, Wittchen $\mathrm{H}-\mathrm{U}$, Kendler KS: Lifetime and 12-month prevalence of DSM-III-R psychiatric disorders in the United States: results from the National Comorbidity Survey. Arch Gen Psychiatry 1994; 51:8-19

4. Bijl RV, Van Zessen G, Ravelli A: Psychiatrische morbiditeit onder volwassenen in Nederland: het NEMESIS-onderzoek, II: prevalentie van psychiatrische stoornissen [Psychiatric morbidity among adults in the Netherlands: the NEMESIS study, II: prevalence of psychiatric disorders]. Ned Tijdschr Geneeskd 1997; 141:2453-2460

5. Wittchen $\mathrm{H}-\mathrm{U}$, Lieb R, Schuster P, Oldehinkel AJ: When is onset? investigations into early developmental stages of anxiety and depressive disorders, in Childhood Onset of "Adult" Psychopathology: Clinical and Research Advances. Edited by Rapoport JL. Washington, DC, American Psychiatric Press, 2000, pp 259302

6. Lewinsohn PM, Rohde P, Seeley JR: Major depressive disorder in older adolescents: prevalence, risk factors and clinical implications. Clin Psychol Rev 1998; 18:765-794

7. Wittchen $\mathrm{H}-\mathrm{U}$, Kessler RC, Pfister $\mathrm{H}$, Lieb M: Why do people with anxiety disorders become depressed? a prospective-longitudinal community study. Acta Psychiatr Scand Suppl 2000; 406: 14-23

8. Caspi A, Moffitt TE, Newman DL, Silva PA: Behavioral observations at age 3 years predict adult psychiatric disorders: Iongitu- dinal evidence from a birth cohort. Arch Gen Psychiatry 1996; 53:1033-1039

9. Hofstra MB, van der Ende J, Verhulst FC: Child and adolescent problems predict DSM-IV disorders in adulthood: a 14-year follow-up of a Dutch epidemiological sample. J Am Acad Child Adolesc Psychiatry 2002; 41:182-189

10. Willett JB, Singer JD, Martin NC: The design and analysis of longitudinal studies of development and psychopathology in context: statistical models and methodological recommendations. Dev Psychopathol 1998; 10:395-426

11. Achenbach TM: Manual for the Child Behavior Checklist/4-18 and 1991 Profile. Burlington, University of Vermont, Department of Psychiatry, 1991

12. Verhulst FC, Akkerhuis GW, Althaus M: Mental health in Dutch children, I: a cross-cultural comparison. Acta Psychiatr Scand Suppl 1985; 323:1-108

13. Verhulst FC, Berden GFMG, Sanders-Woudstra JAR: Mental health in Dutch children, II: the prevalence of psychiatric disorder and relationship between measures. Acta Psychiatr Scand Suppl 1985; 324:1-45

14. Verhulst FC, van der Ende J, Koot HM: Handleiding voor de CBCL/4-18 [Manual for the CBCL/4-18]. Rotterdam, the Netherlands, Erasmus University, Department of Child and Adolescent Psychiatry, Sophia Children's Hospital, 1996

15. Andrews G, Peters L: The psychometric properties of the Composite International Diagnostic Interview. Soc Psychiatry Psychiatr Epidemiol 1998; 33:80-88

16. Van Westerlaak JH, Kropman JA, Collaris JWM: Beroepenklapper [Manual for Occupational Level]. Nijmegen, the Netherlands, Instituut voor Sociologie, 1975

17. Cox DR: Regression models and life tables. J R Stat Soc 1972; 34:187-202

18. Singer JD, Willett JB: Modeling the days of our lives: using survival analysis when designing and analyzing longitudinal studies of duration and timing of events. Psychol Bull 1991; 110: 268-290

19. SPSS 9.0 for Windows. Chicago, SPSS, 1998

20. Cyranowski JM, Frank E, Young E, Shear MK: Adolescent onset of the gender difference in lifetime rates of major depression: a theoretical model. Arch Gen Psychiatry 2000; 57:21-27

21. Petersen AC, Sarigiani PA, Kennedy RE: Adolescent depression: why more girls? J Youth Adolesc 1991; 20:247-271

22. Achenbach TM: Empirically Based Taxonomy: How to Use Syndromes and Profile Types Derived From the CBCL/4-18, TRF, and YSR. Burlington, University of Vermont, Department of Psychiatry, 1993

23. Salmon G, James A, Smith DM: Bullying in schools: self reported anxiety, depression, and self esteem in secondary school children. Br Med J 1998; 317:924-925

24. Simon GE, VonKorff M: Recall of psychiatric history in cross-sectional surveys: implications for epidemiologic research. Epidemiol Rev 1995; 17:221-227

25. Boyer P: Do anxiety and depression have a common pathophysiological mechanism? Acta Psychiatr Scand Suppl 2000; 406:24-29 